\title{
Exploring Outcome Predictors in UK Veterans Treated for PTSD
}

\author{
Dominic Murphy, Walter Busuttil \\ Combat Stress, Leatherhead, UK
}

\begin{abstract}
Post traumatic stress disorder (PTSD) in ex-service personnel is associated with high levels of co-morbidity and significant functional impairment. This paper reports on predictors of treatment outcomes in UK veterans with PTSD. The sample for the study consisted of 244 participants who had received a standardised six week residential treatment programme for PTSD. The programme consisted of individual trauma-focused CBT and group therapy sessions. In this paper, evidence is presented that shows improvements in intrusive, avoidance and hyper-arousal PTSD symptoms six months after the end of treatment; with post-treatment avoidance PTSD symptoms showing the most improvements. The findings suggest that higher levels of baseline anxiety and dissociation are associated with worse post-treatment PTSD outcomes. This suggests the importance of treating these difficulties in UK veterans before intervening for symptoms of PTSD.
\end{abstract}

Keywords: PTSD, military, veterans, treatment, predictors, anxiety

\section{Introduction}

Symptoms of PTSD in military personnel have been associated with a high burden of impairment across a range of psychological, functional, and social exclusion measures (Iversen et al., 2011; Iversen et al., 2009). It has been observed that ex-service personnel with untreated PTSD are at particular risk (Iversen et al., 2005) and that veterans who have been diagnosed with PTSD have poorer outcomes following treatment than non-military peers with PTSD (Watts, Schnurr, Mayo, Young-Xu, Weeks, \& Friedman, 2013; Bradley, Greene, Russ, Dutra, $\&$ Weston, 2005).

Given this, it is encouraging that treatment programmes for PTSD in UK, US, Canadian, and Australian veterans that incorporate elements of individual trauma-focused therapy, and in many cases are supplemented by group therapy, have reported significant improvements that have been maintained at follow up (Richardson, Contractor, Armour, St Cyr, Elhai, \& Sareen, 2014; Forbes, Lewis, Parslow, Hawthorne, \& Creamer, 2008a; Creamer, Morris, Biddle, \& Elliot, 1999; Murphy et al., 2015; Morland et al., 2014; Chard, Schumm, Owens, \& Cottingham, 2010; Resick, Monson, \& Chard, 2007; Monson, Schnurr, Resick, Friedman, Young-Xu, \& Stevens, 2006).

The picture from US studies is mixed with older programmes that had less emphasis on individual trauma therapy and a greater reliance on group sessions demonstrating significant improvements but that these are not always maintained at follow up (Johnson et al., 1996; Goodson \& Helstrom, 2011; Currier, Holland, \& 
Drescher, 2014; Hammarberg \& Silver, 1994; Fontana \& Rosenheck, 1997). More recently, US researchers have demonstrated the efficacy of using Cognitive Processing Therapy (CPT) for veterans with PTSD (Morland et al., 2014; Chard, Schumm, Owens, \& Cottingham, 2010; Monson et al., 2006).

Whilst there are clear differences between culture and the experiences of ex-service personnel from different countries, there does seem to be some evidence that suggests treatment formats that include individual therapy, or a combination of individual and group therapy, are more effective that those that offer group only. A conclusion supported by a recently published meta-analysis that explored the efficacy of treatments offered to veterans with PTSD (Haagen, Smid, Knipscheer, \& Kleber, 2015). In turn, this is supported by systematic review articles that have reported positive treatment outcomes in veterans with PTSD when treated with therapies that include individual cognitive trauma therapy (Bisson, Ehlers, Matthews, Pilling, Richards, \& Turner, 2007; Kitchiner, Roberts, Wilcox, \& Bisson, 2012; Bisson, Roberts, Andrew, Cooper, \& Lewis, 2013).

In addition to understanding which formats of intervention offer the greater benefit in terms of PTSD symptom reduction, elucidating the factors that might present barriers to veterans profiting from therapy is also important to improve treatment effect sizes. It has been argued that military traumas are more complex than single incident traumas (Price, Gros, Strachan, Ruggiero, \& Acierno, 2013) and that military-related traumas are likely to include repeated exposure at high levels of intensity which could to decrease the likelihood of effective treatment (Pietrzak, Whealin, Stotzer, Goldstein, \& Southwich, 2011). Evidence has been presented that suggests that the presence of co-morbid mental health difficulties and PTSD severity (Belsher, Tiet, Garvert, \& Rosen, 2012) negatively predict positive treatment outcomes. In particular, co-morbid symptoms of depression (Richardson et al., 2014; Currier et al., 2014), problems with anger (Owens, Chard, \& Cox, 2008; Forbes, Parslow, Creamer, Allen, McHugh, \& Hopwood, 2008b) and alcohol use (Forbes et al., 2008b) have been shown to predict worse treatment outcomes in veterans with PTSD.

To date there is no research that has explored treatment predictors in UK ex-service personnel. Exploring whether similar factors as above predict treatment outcomes for UK veterans could help service providers adapt interventions to better meet the needs of UK veterans with PTSD.

We have previously reported positive outcomes following treatment at six month follow up. The aim of this paper was to understand the factors that predict treatment outcomes for UK veterans. In this paper we report on whether pre-treatment co-morbid mental health difficulties predict outcomes in UK veterans treated for PTSD. In addition, we describe the longitudinal impact of treatment on avoidance, intrusive and hyper-arousal PTSD symptoms six months after treatment.

\section{Methods}

\section{Setting}

The study employed a within subject design and participants were recruited from UK veterans who had enrolled for a treatment programme for PTSD at Combat Stress. Combat Stress is a mental health charity that provides clinical services for UK military veterans. It has been commissioned by the UK Department of Health as a national specialist service to treat veterans with PTSD (Combat Stress, 2013) and is recognised as a care pathway for veterans with PTSD by the UK's National Health Service. The treatment offered by Combat Stress involved a six week residential programme. During treatment, participants were assigned to a cohort of eight, and treatment involved a mixture of 55 group sessions and 18, 90 minute individual trauma-focused cognitive behaviour therapy sessions. Group sessions included psycho-educational groups (for example, learning a 
psychological understanding of PTSD or the impact of PTSD on communication styles and relationships) or symptoms management groups (for example, managing low mood or grounding strategies for intrusive PSTD symptoms). Further details about the treatment programme have been published elsewhere (Murphy et al., 2015).

\section{Participants}

Inclusion criteria for the treatment programme included being a veteran, having a diagnosis of PTSD and being exposed to a trauma that was related to service life. Exclusion criteria included being actively suicidal, being actively dependent on alcohol, having a diagnosis of a personality disorder or being actively psychotic.

Participants were recruited from individuals who had enrolled for treatment between late 2012 and mid 2014 and had been successfully followed up six months later. During this time period, 312 individuals were offered treatment. Of these, 292/312 (94\%) completed treatment. Completing treatment was defined as finishing a minimum of five weeks of the six week course and at least 15 individuals TF-CBT sessions. The most common reason for not completing treatment was experiencing physical health problems during the programme. In addition, six individuals were deemed unsuitable for therapy by the clinical team and five individuals left the programme due to problems related to alcohol. 244/292 (84\%) were successfully followed up six months after treatment and are the sample used for the current study. In a previous publication, we demonstrated that there were no significant differences on a range of health outcomes between individuals who did not complete the treatment and those who did and also between individuals that were followed up successfully at six months and those lost to follow up (Murphy et al., 2015). Based on these findings, we were confident that there was no evidence for the presence of a health bias between individuals we were able to follow up or not.

\section{Measures}

At admission participants were asked to complete a questionnaire. This included questions on socio-demographic characteristics such as age, sex, relationship status, employment status and educational achievement. In addition, information about military history was recorded. This included data about the service they had been in (Army, Navy or Royal Air Force), their last rank (officer or other ranks), their main role in service (combat or non combat), how they left the Armed Forces (voluntarily or non-voluntarily), time since leaving the military and where they had deployed. A range of health outcomes were collected. These health outcomes were repeated at discharge, and at a six week and six month follow up.

\section{Primary Ouztcome Measures}

For this study, our primary health outcome was a measures for PTSD. We measured symptoms of PTSD using the PTSD Symptom Scale Interview (PSS-I). This is a structured interview schedule that was completed by the clinician working with the participant. The PSS-I contains 17 items and assess the presence and severity of symptoms of PTSD (Foa, Riggs, Dancu, \& Rothbaum, 1993). The 17 items map onto the three clusters of diagnostic symptoms for PTSD from the DSM-IV (APA, 2005). As such, the PSS-I provides information not only on overall PTSD severity, but also data about these three clusters: intrusion, avoidance and hyper-arousal symptoms.

\section{Secondary Outcomes}

These included a range of co-morbid presentations. Symptoms of depression were assessed using the Patient Health Question (PHQ-9) and participants met case criteria if they scored 10 or above (Titov, Dear, 
McMillan, Anderson, Zou, \& Sunderland, 2011; Cameron, Crawford, Lawton, \& Reid, 2008; Kroenke \& Spitzer, 2002); anxiety was measured using the Generalised Anxiety Disorder assessment (GAD-7) and participants who scored eight or above were deemed as meeting case criteria (Swinson, 2006); difficulties with anger were explored using the five item Dimension of Anger Reactions (DAR-5) and to meet case criteria participants had to score 12 or above (Forbes et al., 2004; Forbes et al., 2014); symptoms of dissociation were explored with the Dissociative Experiences Scale (DES-II) and meeting case criteria was defined as a score of 20 or above (Carlson \& Putnam, 1993; Van Ijzendoorn \& Schuengal, 1996); Alcohol difficulties were assessed using the Alcohol Use Disorders Identification Test (AUDIT) and we have used eight or more to define hazardous drinking (Babor, Higgins-Biddle, Saunders, \& Monteiro, 2001). Perceived impaired functioning was recorded using the Work and Social Adjustment Scale (WSAS) (Mundt, Marks, Shear, \& Greist, 2002). This is a self reported measure and contains five item exploring different aspects functioning. These are ability to work, home management, being able to engage in social activities, being able to engage in private leisure activities and being able to maintain personal relationships.

\section{Analysis}

The first stage of the analysis was to explore the socio-demographic characteristics and military service information for the sample. In addition, a variable was constructed. This variable calculated changes in PSS-I scores between baseline and six month follow up. Changes in PSS-I score were then assigned to four groups (no change or an increase in score, a reduction of score between one and nine points, a reduction of score between 10 and 19 points and a reduction in score of over 20 points).

Univariable Regression models were fitted to look at the relationships between PSS-I scores at six months post treatment and baseline secondary health outcomes. Three different multivariable regression models were then fitted for each health outcome which built upon each other in a stepwise fashion. The first adjusted for age and employment status, the second further added pre-treatment functional impairment (WSAS) and the third added severity of pre-treatment PTSD (PSS-I scores).

The next stage of the analysis was to fit random slopes growth models to explore longitudinal changes in the PSS-I subscales scores between admission, discharge, six week and six month follow up. The random slope growth models fitted were nonlinear and used a fixed coefficient of time squared (Steele, 2008). Models were adjusted for employment status and age group $(<35,35-44$ and $>45)$. This was because we found these to significantly improve the fit of the models using likelihood ratio tests. The final stage of the analysis was to repeat the above regression models that had been used to explore the impact of baseline health on six month post treatment PSS-I scores, but this time to explore the relationships between baseline health on six month post-treatment PSS-I subscales scores (e.g., for intrusive, avoidance and hyper-arousal symptoms). This final set of analyses were restricted to only the pre-treatment health outcomes that had previous been found to be associated with worse post-treatment PTSD. Analyses were conducted using Stata13 (StataCorp, College Station, TX).

\section{Results}

Our sample consisted of 244 participants. The majority of the sample were male (99\%), $24 \%$ of the sample were aged less than $35,35 \%$ between the ages of 35 and 44 and $41 \%$ aged over $45,91 \%$ were educated to $O$ levels or below and $9 \%$ educated to A Levels or above, $53 \%$ were in a relationship and $47 \%$ single and $37 \%$ 
reported working in either a part time or full time capacity and $63 \%$ were not working. Table 1 describes the military history of the sample. The majority were in the Army, had been in combat roles during their military service and lower ranks. $60 \%$ of the sample reported having left the services voluntarily versus $40 \%$ who reported having left the Armed Forces against their will. $40 \%$ of the sample reported having left the military within the last nine years; the remaining $60 \%$ reported having left the military 10 or more years previously. The majority of our sample reported having deployed to Northern Ireland, participants who had deployed to Iraq since 2003 were the next largest group and then similar numbers of participants endorsed having deployed to either the Falkland conflict, the 1991 Gulf war, to Bosnia or Kosovo during the 1990s and Afghanistan since 2001. It was observed that $86.5 \%$ of the sample showed improvements in their PTSD symptoms. Whilst not presented, no significant associations were found between socio-demographic or military characteristics and PTSD treatment outcomes scores on the PSS-I.

Table 1

Military Service Information and Change in PTSD Scores

\begin{tabular}{|c|c|}
\hline & $\begin{array}{l}\text { Number }{ }^{1}(n=244) \\
n(\%)\end{array}$ \\
\hline \multicolumn{2}{|l|}{ Service } \\
\hline Royal Navy & $20(8.3)$ \\
\hline Army & $207(85.5)$ \\
\hline Royal Air Force & $15(6.2)$ \\
\hline \multicolumn{2}{|l|}{ Role in military } \\
\hline Non-combat & $49(20.4)$ \\
\hline Combat & $191(79.6)$ \\
\hline \multicolumn{2}{|l|}{ Last rank } \\
\hline Officer & $13(5.4)$ \\
\hline Other ranks & $229(94.6)$ \\
\hline \multicolumn{2}{|l|}{ Type of discharge from military } \\
\hline Voluntary & $141(60.3)$ \\
\hline Non-voluntary & $93(39.7)$ \\
\hline \multicolumn{2}{|l|}{ Years since left the military } \\
\hline $1-9$ & $96(39.5)$ \\
\hline $10-19$ & $71(29.2)$ \\
\hline $20+$ & $76(31.3)$ \\
\hline \multicolumn{2}{|l|}{ Areas of deployment ${ }^{2}$} \\
\hline Falklands Conflict & $36(14.8)$ \\
\hline Northern Ireland & $137(56.2)$ \\
\hline 1991 Gulf War & $41(16.8)$ \\
\hline Bosnia and Kosovo & $55(22.5)$ \\
\hline Afghanistan since 2001 & $42(17.2)$ \\
\hline Iraq since 2003 & $80(32.8)$ \\
\hline \multicolumn{2}{|c|}{ Changes in PSS-I scores at 6 month F/U } \\
\hline Score stayed the same or got worse & $33(13.5)$ \\
\hline Score improved 1-9 points & $66(27.0)$ \\
\hline Score improved 10-19 points & $72(29.5)$ \\
\hline Score improved 20 or more points & $73(30.0)$ \\
\hline
\end{tabular}

Notes. ${ }^{1}$ Numbers may not add up to 244 because of missing data $;{ }^{2}$ Individuals may have deployed to multiple locations. 
In Table 2, regression models were fitted to report the relationships between baseline co-morbid health outcomes and six month follow up PTSD PSS-I scores. In the unadjusted models, meeting case criteria for baseline generalised anxiety and dissociation was significantly associated with higher PSS-I scores at follow up. Meeting case criteria for baseline generalised anxiety predicted the highest increase in PSS-I score post-treatment $(\beta=14.5)$. The first model adjusted for age and employment status; this led to an increase in effect size for baseline anxiety and a reduction for dissociation. The second model further adjusted for pre-treatment self-reported functional impairment. Both high levels of pre-treatment anxiety and dissociation remained associated with worse post-treatment PTSD outcomes. Model three adjusted for pre-treatment PTSD severity (PSS-I scores). This resulted in a reduced effect size for pre-treatment anxiety and no significant associated between pre-treatment dissociation and PTSD treatment outcomes.

Table 2

Relationships Between Baseline Health Outcomes and Post-Treatment PTSD Scores

\begin{tabular}{lcccc}
\hline & Unadjusted $\beta(95 \% \mathrm{CI})$ & \multicolumn{3}{c}{ Adjusted $\beta(95 \% \mathrm{CI})$} \\
\cline { 3 - 5 } & & Model 1 & Model 2 & Model 3 \\
\hline Depression (PHQ-9) & $4.53(-0.63,9.70)$ & $4.33(-0.97,9.64)$ & $1.46(-3.98,6.89)$ & $-0.96(-6.61,4.39)$ \\
Generalised anxiety (GAD-7) & $14.5(7.06,21.9)^{*}$ & $15.4(7.63,23.3)^{*}$ & $14.6(6.57,22.7)^{*}$ & $9.89(1.49,18.3)^{*}$ \\
Anger (DAR-5) & $2.31(-0.73,5.35)$ & $1.75(-1.44,4.94)$ & $0.10(-3.13,3.33)$ & $-0.24(-3.36,2.87)$ \\
Alcohol (AUDIT) & $2.89(-0.16,5.94)$ & $2.64(-0.51,5.80)$ & $2.91(-0.16,5.97)$ & $2.48(-0.48,5.43)$ \\
Dissociation (DES-II) & $6.05(2.94,9.16)^{*}$ & $5.78(2.52,9.04)^{*}$ & $4.36(1.04,7.69)^{*}$ & $2.49(-0.90,5.86)$ \\
\hline
\end{tabular}

Note. $^{*}=p \leq 0.05$; Model $1 \beta$ adjusted for age and employment status; Model 2 additionally adjusted for pre-treatment functional impairment (WSAS); Model 3 in addition to model 2, adjusted for pre-treatment PSS-I score.

Table 3

Changes on PTSD Subscales Post-Treatment

\begin{tabular}{lllll}
\hline & $\begin{array}{l}\text { Admission } \\
\text { mean score }\end{array}$ & $\begin{array}{l}6 \text { month f/u } \\
\text { mean score }\end{array}$ & $\begin{array}{l}\text { Unadjusted model } \\
\beta(95 \% \mathrm{CI})\end{array}$ & $\begin{array}{l}\text { Adjusted model } \\
\beta(95 \% \mathrm{CI})\end{array}$ \\
\hline PSS-I Subscale & & & & \\
\hline Intrusions & 10.1 & 6.80 & $-2.74(-3.18,-2.30)^{*}$ & $-2.69(-3.14,-2.24)^{*}$ \\
Avoidance & 14.9 & 8.86 & $-6.49(-7.13,-5.85)^{*}$ & $-6.61(-7.27,-5.95)^{*}$ \\
Hyper-arousal & 12.2 & 8.60 & $-3.69(-4.14,-3.24)^{*}$ & $-3.69(-4.14,-3.23)^{*}$ \\
\hline
\end{tabular}

Notes. ${ }^{*}=p \leq 0.05$; Adjusted model $\beta$ adjusted for age and employment status.

Table 3 describes longitudinal changes in the three PSS-I subscales following treatment. Significant improvements in all three of the PSS-I subscales were observed. Larger reductions in avoidance PTSD scores were noted compared to reductions in intrusion and hyper-arousal scores. The analyses from Table 2 were repeated in Table 4 to explore how baseline health predicted changes on the PSS-I subscales post-treatment. Analyses were restricted to only those health outcomes that were found to be significant in Table 2. In the unadjusted regression model, meeting case criteria at baseline for anxiety and dissociation predicted worse outcomes across all three of the subscales on the PSS-I. In particular, baseline anxiety appeared to predict a worsening of avoidance symptoms and baseline dissociation a worsening of the intrusive symptoms on the PSS-I. Model one adjusted for age and employment status. The associations remain significant with effect sizes and for pre-treatment anxiety increasing marginally and those for dissociation reducing. Model 2 further adjusted for pre-treatment self-reported functional impairment. Associations between anxiety and PTSD 
outcomes across the three subscales remain significant. Pre-treatment dissociation remained significantly associated with higher post-treatment scores on the intrusive and hyper-arousal PSS-I subscales. Model 3 further adjusted for pre-treatment PTSD severity. Associations between pre-treatment anxiety and higher post-treatment scores across all three of the PSS-I subscales remained significant. However, higher levels of pre-treatment dissociation was no longer significantly associated with higher scores on any of the three subscales.

Table 4

Relationships Between Baseline Health Outcomes and Post-Treatment PTSD PSS-I Subscale Scores

\begin{tabular}{|c|c|c|c|c|}
\hline & \multirow{2}{*}{ Unadjusted $\beta(95 \% \mathrm{CI})$} & \multicolumn{3}{|c|}{ Adjusted $\beta(95 \% \mathrm{CI})$} \\
\hline & & Model 1 & Model 2 & Model 3 \\
\hline \multicolumn{5}{|l|}{ Intrusion subscale } \\
\hline Generalised anxiety (GAD-7) & $3.60(0.34,6.87)^{*}$ & $3.76(0.26,7.25)^{*}$ & $3.91(0.22,7.60)^{*}$ & $1.18(-2.60,4.97)^{3}$ \\
\hline Dissociation (DES-II) & $2.52(1.16,3.87)^{*}$ & $2.34(0.91,3.78)^{*}$ & $2.11(0.62,3.60)^{*}$ & $1.20(-0.31,2.70)$ \\
\hline \multicolumn{5}{|l|}{ Avoidance subscale } \\
\hline Generalised anxiety (GAD-7) & $6.24(2.82,9.65)^{*}$ & $6.55(2.97,10.1)^{*}$ & $6.01(2.30,9.72)^{*}$ & $4.51(0.59,8.43)^{*}$ \\
\hline Dissociation (DES-II) & $2.02(0.56,3.48)^{*}$ & $1.80(0.28,3.33)^{*}$ & $1.15(-0.39,2.69)$ & $0.47(-1.11,2.05)$ \\
\hline \multicolumn{5}{|l|}{ Hyper-arousal subscale } \\
\hline Generalised anxiety (GAD-7) & $4.89(2.54,7.25)^{*}$ & $5.31(2.86,7.76)^{*}$ & $5.02(2.47,7.56)^{*}$ & $3.80(1.13,6.47)^{*}$ \\
\hline Dissociation (DES-II) & $1.69(0.69,2.69)^{*}$ & $1.73(0.70,2.76)^{*}$ & $1.27(0.22,2.32)^{*}$ & $0.75(-0.33,1.82)$ \\
\hline
\end{tabular}

Notes. ${ }^{*}=p \leq 0.05$; Model $1 \beta$ adjusted for age and employment status; Model 2 additionally adjusted for pre-treatment functional impairment (WSAS); Model 3 in addition to model 2, adjusted for pre-treatment PSS-I score.

\section{Discussion}

We have previously reported significant improvements on a range on mental health outcomes following treatment for PTSD in UK veterans (Murphy et al., 2015). In this paper, we focused on analysing the subscales that make up the PSS-I. Significant improvements were observed in all three of the DSM-IV PTSD symptom clusters (intrusive, avoidance, and hyper-arousal symptoms). We found the largest reductions in PSS-I scores were for symptoms within the avoidance subscale. Given that TF-CBT aims to support individuals to drop avoidance strategies and relive aspects of their traumas, significant reductions in this domain are maybe not surprising. It is encouraging to observe that these reductions are enduring at six month follow up.

In this paper, we also reported on the impact of baseline co-morbid mental health difficulties on PTSD symptoms following treatment for PTSD in veterans of the UK Armed Forces. We found that individuals who reported higher levels of baseline generalised anxiety and dissociation reported worse post-treatment PTSD outcomes than their counterparts. Of these two co-morbid health outcomes, the reported effect sizes on PTSD treatment outcomes were larger for anxiety than dissociation. These relationships remain significant after adjustment for functional impairment. After further adjustment for pre-treatment PTSD severity only baseline anxiety remained a significant predictor of post-treatment PTSD severity. Taken together this suggests that for the current sample of UK veterans, within the health outcomes investigated, a higher level of pre-treatment anxiety was the strongest predictor of PTSD treatment outcomes.

We found that despite higher rates of co-morbidity veterans with military-related PTSD can make significant gains post-treatment. This finding has been noted in other veteran populations around the world (Richardson, Eihai, \& Sareen, 2011; Forbes et al., 2008a; Morland et al., 2014; Chard et al., 2010; Monson et al., 
2006). Differences in how co-morbid mental health difficulties may affect treatment have differed between populations. Canadian researchers have reported that co-morbid presentations did not predict treatment outcome within a sample of 102 veterans (Richardson et al., 2011). Further research within a different larger sample of Canadian veterans did observe a relationship between symptoms of depression severity and PTSD treatment outcomes (Richardson et al., 2014). A similar pattern has been observed in US veterans undergoing treatment for PTSD with individuals with lower levels of baseline mental health having better outcomes (Currier et al., 2014; Hammarberg \& Silver, 1994). The findings presented within the current study that suggest a different pattern for UK veterans with generalised anxiety and dissociative symptoms predicting changes in PTSD scores post-treatment. The role of dissociation is intriguing and is supported by theoretical assumptions about there being a dissociative subtype of PTSD (Wolf, Lunney, Miller, Resick, Friedman, \& Schnurr, 2012; Wolf, 2013; Lanius, Brand, Vermetten, Frewen, \& Spiegel, 2012). However, this finding contrasts with recent research, not restricted to military samples, that appears to suggest that dissociation does not predict treatment outcomes (Halvorsen, Stenmark, Neuner, \& Nordahl, 2014; Cloitre, Petkova, Wang, \& Lu, 2012; Hagenaars, van Minnen, $\&$ Hoogduin, 2010). Whilst differences exist between the findings of these studies, it appears that higher levels of co-morbid mental health difficulties predict worse treatment outcomes for symptoms of PTSD in veterans. This suggests the importance of treating co-morbidities before treating symptoms PTSD.

In common with previous research, we found no evidence that alcohol difficulties nor number of years with PTSD symptoms predicted treatment outcomes (Richardson et al., 2014). This may have been an artefact of our exclusion criteria that participants were not actively dependent on alcohol at the start of treatment which could have led to excluding individuals with alcohol problems and hence explain this finding. However, even with this exclusion criterion the mean pre-treatment score for the study sample on the AUDIT was 8.93 (95\% CI 7.85, 10.0) which indicates hazardous drinking levels rather than alcohol dependence and suggests that problems with alcohol were still present within the study sample.

We found that over $60 \%$ of participants reported having left the military at least 10 years previous to engaging in treatment with Combat Stress. We were unable to record the time since an individual was exposed to their index trauma, but because an inclusion criterion was exposure to a military trauma, time since leaving the Forces could be considered a proxy measure for time since trauma. Indeed, if this was the case it may be conservative proxy measure because a participant's index trauma may have occurred at any time during their military career's rather than at the end, so the actual length of time that individuals may have been experiencing symptoms of PTSD may be longer. As such, whilst limitations exist, this suggests that within this population of UK military veterans significant periods of time have past between an individual's being exposed to a trauma and seeking support. This is supported by previous research that reported that over $70 \%$ of veterans who received treatment for PTSD from Combat Stress reported that it was the first time they had been able to engage in clinical services (Murphy et al., 2015). Given the length of time between leaving the Armed Forces and receiving treatment discussed above, it is encouraging that treatment gains were observed. This is because it suggests that individuals who have suffered from symptoms of PTSD for considerable periods of time are just as likely to profit from treatment as their counterparts who sought help sooner. A relationship has been observed between poorer physical health and worse PTSD treatment outcomes in US veterans (Currier et al., 2014). Given this, an area for future research would be to explore the relationship between physical health and mental health difficulties in UK veterans. 


\section{Strengths and Limitations}

The current study profited from recruiting participants who had enrolled on the same treatment programme. The treatment programme should have good treatment fidelity because it has been manualised and was offered as a standardised package to all veterans. We were able to follow up over $80 \%$ of those offered the treatment at six months. This compares favourably with similar PTSD treatment programmes offered to military veterans in different countries (Forbes et al., 2008a; Richardson et al., 2011; Goodson \& Helstrom, 2011; Currier et al., 2014). Further, we are confident that no differences were present between individuals that it was possible to review at six months and those lost to follow up (Murphy et al., 2015).

A limitation to the current study was that a within subject design was employed rather than a randomised controlled trial (RCT) design. This was because we had been funded by the Department of Health to provide a timely national clinical service available for all UK military veterans with PTSD, which was to be based upon existing NICE guidance (NICE, 2005) and the published experiences of the Australian Veteran Affairs PTSD treatment programmes (Forbes et al., 2008a; Creamer, Forbes, Biddle, \& Elliot, 2002; Creamer et al., 1999) rather than run an RCT. However, this does mean that limitations exist as to whether inferences about causality that can be made. For example, because we did not take a randomised sample there could be confounding variables that were unmeasured that have introduced bias into our findings. When comparing research into treatment outcomes from different countries it is worth noting that these also did not employ RCT designs. This means that limitations may also exist in the conclusions drawn from these studies. The exclusion criteria could have meant that certain subgroups of veterans with PTSD were excluded from the sample, for example, individuals meeting diagnostic criteria for both PTSD and a personality disorder.

\section{Conclusions}

We provide evidence that describes an association between higher levels of pre-treatment generalised anxiety and dissociation and worse post-treatment outcomes in terms of PTSD severity. We observed significant improvement in clusters of avoidance, intrusive and hyper-arousal PTSD symptoms and that overall, the vast majority of the participants had a reduction in PTSD symptoms severity six months after treatment. Our findings suggest the importance of treating co-morbid symptoms of generalised anxiety and dissociation within veterans with PTSD. More research is needed to explore how best to treat these co-morbidities and then the subsequent impact on outcomes following future treatment for PTSD.

\section{Reference}

APA. (2005). Quick Reference to the diagnostic criteria from DSM-IV. Washington, D.C.: American Psychiatric Association.

Babor, T. F., Higgins-Biddle, J. C., Saunders, J. B., \& Monteiro, M. G. (2001). AUDIT: The alcohol use disorders identification test. Geneva: Department of Mental Health and Substance Dependence, World Health Organization.

Belsher, B., Tiet, Q., Garvert, D., \& Rosen, C. (2012). Compensation and treatment: Disability benefits and outcomes of US veterans receiving residential PTSD treatment. Journal of Traumatic Stress, 25, 494-502.

Bisson, J., Ehlers, A., Matthews, R., Pilling, S., Richards, D., \& Turner, S. (2007). Psychological treatments for chronic post-traumatic stress disorder: Systematic review and meta-analysis. British Journal of Psychiatry, 190, 97-104.

Bisson, J., Roberts, N., Andrew, M., Cooper, R., \& Lewis, C. (2013). Psychological therapies for chronic post-traumatic stress disorder (PTSD) in adults (12). Cochrane Database of Systemic Reviews. doi:10.1002/14651858.CD003388.pub4

Bradley, R., Greene, J., Russ, E., Dutra, L., \& Weston, D. (2005). A multi-dimensional meta-analysis pf psychotherapy for PTSD. American Journal of Psychiatry, 162, 214-227.

Cameron, I. M., Crawford, J. R., Lawton, K., \& Reid, I. C. (2008). Psychometric comparison of PHQ-9 and HADS for measuring depression severity in primary care. British Journal of General Practice, 58(546), 32-36. 
Carlson, E., \& Putnam, F. (1993). An update on the dissociative experiences scale. Dissociation, 6, 16-27.

Chard, K., Schumm, J., Owens, G., \& Cottingham, S. (2010). A comparison of OEF and OIF veterans and Vietnam veterans receiving cognitive processing therapy. Journal of Traumatic Stress, 22(1), 11-19.

Cloitre, M., Petkova, E., Wang, J. G., \& Lu, F. (2012). An examination of the infulence of a sequential treatment on the course and impact of dissociation among women with PTSD related to childhood abuse. Depression and Anxiety, 29, $709-717$.

Combat Stress. (2013). Combat Stress 2013 annual review of clinical services. Retrieved from: http://www.combatstress.org.uk/about-us/annual-review/

Creamer, M., Forbes, D., Biddle, D., \& Elliot, P. (2002). Inpatient versus day hospital treatment for chronic, combat-related posttraumatic stress disorder: a naturalistic comparison. The Journal of Nervous and Mental Disease, 190(3), 183-189.

Creamer, M., Morris, P., Biddle, D., \& Elliot, P. (1999). Treatment outcome in Australian veterans with combat-related posttraumatic stress disorder: a cause for cautious optimism? Journal of Traumatic Stress, 12(4), 545-558.

Currier, J., Holland, J., \& Drescher, K. (2014). Residential treatment for combat-related posttraumatic stress disorder: Identifying trajectories of change and predictors of treatment response. PLOS ONE, 9(7), e101741. doi:10.1371/journal.pone.0101741.

Foa, E. B., Riggs, D., Dancu, C., \& Rothbaum, B. O. (1993). Reliability and validity of a brief instrument for assessing post-traumatic stress disorder. Journal of Traumatic Stress, 6, 459-473.

Fontana, A., \& Rosenheck, R. (1997). Effectiveness and cost of the inpatient treatment of posttraumatic stress disorder: Comparison of three models of treatment. American Journal of Psychiatry, 154, 758-765.

Forbes, D., Alkemade, N., Mitchell, D., Elhai, J., McHugh, T., Bates, G. et al. (2014). Utility of the Dimensions of Anger Reactions-5 (DAR-5) scale as a brief anger measure. Depression and Anxiety, 31(2), 166-173.

Forbes, D., Hawthorne, G., Elliot, P., McHugh, T., Biddle, D., Creamer, M. et al. (2004). A concise measure of anger in combat-related posttraumatic stress disorder. Journal of Traumatic Stress, 17(3), 249-256.

Forbes, D., Lewis, V., Parslow, R., Hawthorne, G., \& Creamer, M. (2008a). Naturalistic comparison of models of programmatic interventions for combat-related post-traumatic stress disorder. Australian and New Zealand Journal of Psychiatry, 42, 1051-1059.

Forbes, D., Parslow, R., Creamer, M., Allen, N., McHugh, T., \& Hopwood, M. (2008b). Mechanism of anger and treatment outcome in combat veterans with posttraumatic stress disorder. Journal of Traumatic Stress, 21, 142-149.

Goodson, J., \& Helstrom, A. (2011). Treatment of posttraumatic stress disorder in US combat veterans: a meta-analytic review. Psychological Reports, 109, 573-599.

Haagen, J., Smid, G., Knipscheer, J., \& Kleber, R. (2015). The efficacy of recommended treatments for veterans with PTSD: A metaregression analysis. Clinical Psychology Review. doi:10.1016/j.cpr.2015.06.008

Hagenaars, M., van Minnen, A., \& Hoogduin, K. (2010). The impact of dissociation and depression on the efficacy of prolonged exposure treatment for PTSD. Behaviour Research \& Therapy, 48, 19-27.

Halvorsen, J., Stenmark, H., Neuner, F., \& Nordahl, H. (2014). Does dissociation moderate treatment outcomes of narrative exposure therapy for PTSD? A secondary analysis from a randomized controlled clinical trial. Behaviour Research \& Therapy, 57, 21-28.

Hammarberg, M., \& Silver, S. (1994). Outcome for treatment for post-traumatic stress disorder in a primary care unit serving Vietnam veterans. Journal of Traumatic Stress, 7, 195-216.

Iversen, A., Dyson, C., Smith, N., Greenberg, N., Walwyn, R., Unwin, C. et al. (2005). "Goodbye and good luck": The mental health needs and treatment experiences of British ex-service personnel. British Journal of Psychiatry, 186(June), 480-486.

Iversen, A. C., van, S. L., Hughes, J. H., Browne, T., Hull, L., Hall, J. et al. (2009). The prevalence of common mental disorders and PTSD in the UK military: Using data from a clinical interview-based study. BMC psychiatry, 9, 68.

Iversen, A. C., van, S. L., Hughes, J. H., Greenberg, N., Hotopf, M., Rona, R. J. et al. (2011). The stigma of mental health problems and other barriers to care in the UK Armed Forces. BMC Health Services Research, 11, 31.

Johnson, D., Rosenheck, R., Fontana, A., Lubin, H., Charney, D., \& Southwick, S. M. (1996). Outcome of intensive inpatient treatment for combat-related posttraumatic stress disorder. American Journal of Psychiatry, 153, 771-777.

Kitchiner, N., Roberts, N., Wilcox, D., \& Bisson, J. (2012). Systematic review and meta-analysis of psychosocial interventions for veterans of the military. European Journal of Psychotraumatology, 3. doi: 10.3402/ejpt.v3i0.19267

Kroenke, K., \& Spitzer, R. (2002). The PHQ-9: A new depression diagnostic and severity measure. Psychiatric Annals, 32(9), 509-515.

Lanius, R., Brand, B., Vermetten, E., Frewen, P., \& Spiegel, D. (2012). The dissociative subtype of posttraumatic stress disorder: Rational, clinical amd neurobiological evidence, and implications. Depression and Anxiety, 29, 701-708. 
Monson, C., Schnurr, P., Resick, P., Friedman, M. J., Young-Xu, Y., \& Stevens, S. (2006). Cognitive processing therapy for veterans with military-related PTSD. Journal of Consulting \& Clinical Psychology, 74(5), 898-907.

Morland, L., Mackintosh, M., Greene, C., Rosen, C., Chard, K., Resick, P. et al. (2014). Cognitive processing therapy for posttraumatic stress disorder delivered to rural veterans via telehealth: a randomised noninferiority clinical trial. Journal of Clinical Psychiatry, 75(5), 470-476.

Mundt, J. C., Marks, I. M., Shear, M. K., \& Greist, J. H. (2002). The work and social adjustment scale: A simple measure of impairment in functioning. British Journal of Psychiatry, 180, 461-164.

Murphy, D., Hodgman, G., Carson, C., Spencer-Harper, L., Hinton, M., Wessely, S. et al. (2015). Mental health and functional impairment outcomes following a six week intensive treatment programme for UK military veterans with post traumatic stress disorder (PTSD): A naturalistic study to explore dropout and health outcomes at follow-up. BMJ Open, 5. doi:10.1136/bmjopen-2014-007051.

NICE. (2005). Post-traumatic stress disorder (PTSD): The management of PTSD in adults and children in primary and secondary care: Quick reference guide. London: NICE.

Owens, G., Chard, K., \& Cox, T. (2008). The relationship between maladaptive cognitions, anger expression, and posttraumatic stress disorder among veterans in residential treatment. Journal of Aggression, Maltreatment \& Trauma, 17(439), 452.

Pietrzak, R., Whealin, J. M., Stotzer, R., Goldstein, M., \& Southwich, S. (2011). An examination of the relation between combat experiences and combat-related posttraumatic stress disorder in a sample of Connecticut OEF-OIF veterans. Journal of Psychiatric Research, 45(1579), 1584.

Price, M., Gros, D., Strachan, M., Ruggiero, K., \& Acierno, R. (2013). Combat experiences, pre-deployment training and outcome of exposure therapy for post-traumatic stress disorder in Operation Enduring Freedom/Operation Iraqi Freedom veterans. Clinical Psychology and Psychotherapy, 20, 277-285.

Resick, P., Monson, C., \& Chard, K. (2007). Cognitive processing therapy: Veteran/Military version. Washington, D.C.: Department of Veterans' Affairs.

Richardson, D., Contractor, A., Armour, C., St Cyr, K., Elhai, J., \& Sareen, J. (2014). Predictors of long-term treatment outcome in combat and peacekeeping veterans with military-related PTSD. The Journal of Clinical Psychiatry, 75(11), 1299-1305.

Richardson, D., Eihai, J., \& Sareen, J. (2011). Predictors of treatment response in Canadian combat and peacekeeping veterans with military-related PTSD. Journal of Nervous and Mental Disease, 199(9), 639-645.

Steele, F. (2008). Multilevel models for longitudinal data. Journal of the Royal Statistical Society, Series A, 171, 5-19.

Swinson, R. P. (2006). The GAD-7 scale was accurate for diagnosing generalised anxiety disorder. Evidence Based Medicine, 11(6), 184.

Titov, N., Dear, B. F., McMillan, D., Anderson, T., Zou, J., \& Sunderland, M. (2011). Psychometric comparison of the PHQ-9 and BDI-II for measuring response during treatment of depression. Cognitive Behaviour Therapy, 40(2), 126-136.

Van Ijzendoorn, M., \& Schuengal, C. (1996). The measurement of dissociation in normal and clinical populations: Meta-analytic validation of the Dissociative Experiences Scale. Clinical Psychology Review, 16, 365-382.

Watts, B., Schnurr, P., Mayo, L., Young-Xu, Y., Weeks, W., \& Friedman, M. J. (2013). Meta-analysis of the efficay of treatments for posttraumatic stress disorder. Journal of Clinical Psychiatry, 74, 541-550.

Wolf, E. (2013). The dissociative subtype of PTSD: Rationale, evidence, and future directions. PTSD Research Quarterly, 24, 1-8.

Wolf, E., Lunney, C., Miller, M., Resick, P., Friedman, M. J., \& Schnurr, P. (2012). The dissociative subtype of PTSD: A replication and extension. Depression and Anxiety, 29, 679-688. 